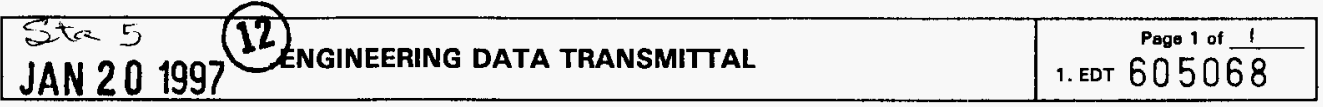

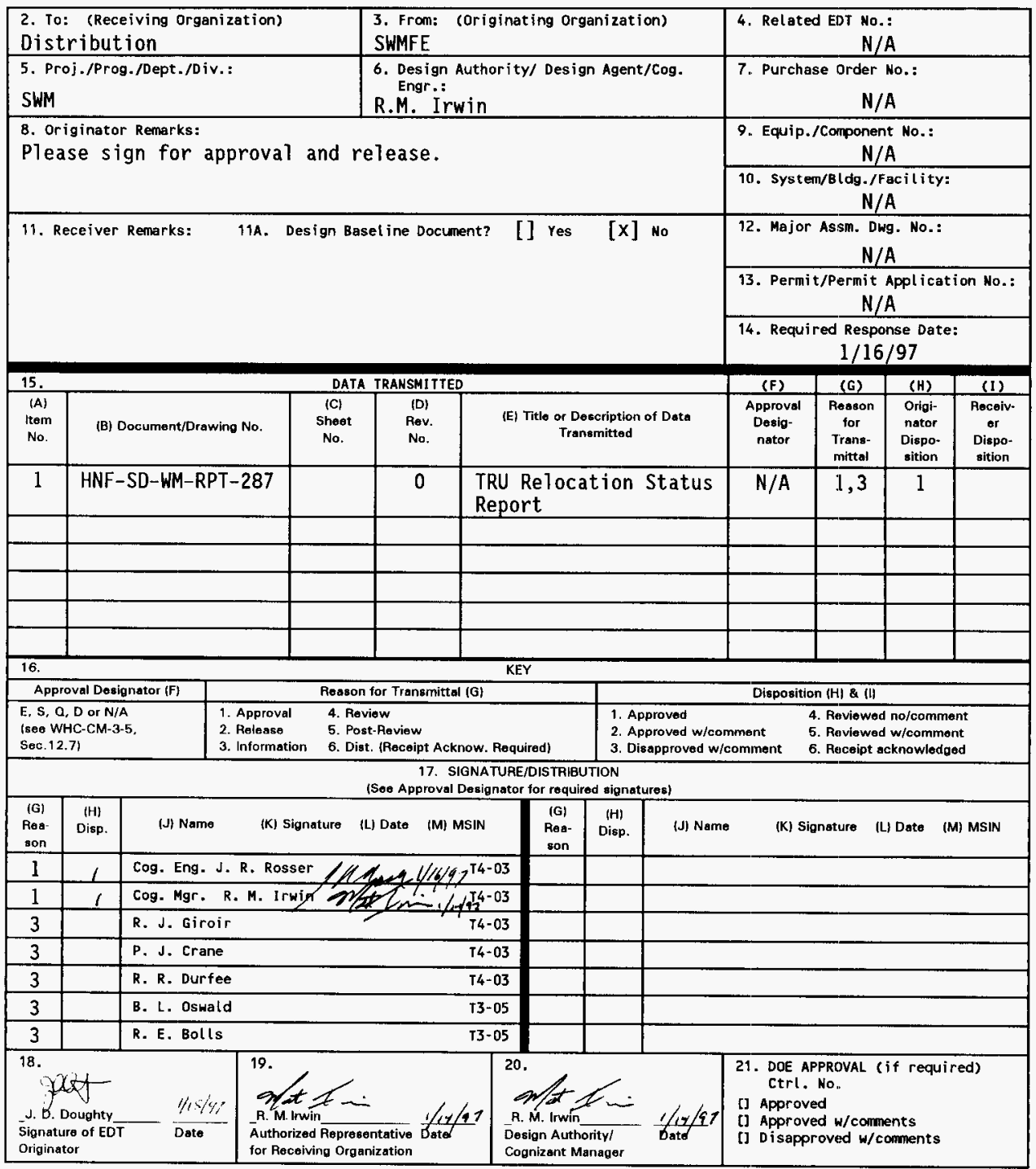




\section{TRU Relocation Status Report}

R. M. Irwin

Rust Federal Services of Hanford. Inc., Richland, WA 99352

U.S. Department of Energy Contract DE-AC06-87RL10930

EDT/ECN: 605068

Org Code: 32540

UC: 510

B\&R Code: EW3130020

Charge Code: $A 31 \mathrm{~J} 3$

Tota1 Pages: 19

Key Words: Transuranic waste, relocation, assay, verification, Low-Level Burial Grounds, TRUSAF. T Plant, Central Waste Complex

Abstract: To meet an internal milestone. 257 containers identified as contact-handled transuranic waste are being relocated from uncovered drum modules in the Low-Level Burial Grounds into covered facilities. The project is not yet complete. This report discusses the project and its status as of December 31, 1996.

TRADEMARK DISCLAIMER. Reference herein to any specific commercial product, process, or service by trade name, trademark, manufacturer, or otherwise, does not necessarily constitute or imply its endorsement, recommendation, or favoring by the United States Government or any agency thereof or its contractors or subcontractors.

Printed in the United States of America. To obtain copies of this document, contact: WHC/BCS Document Control Services, P.O. Box 1970, Mailstop H6-08, Richland WA 99352, Phone (509) 372-2420; Fax (509) 376-4989.
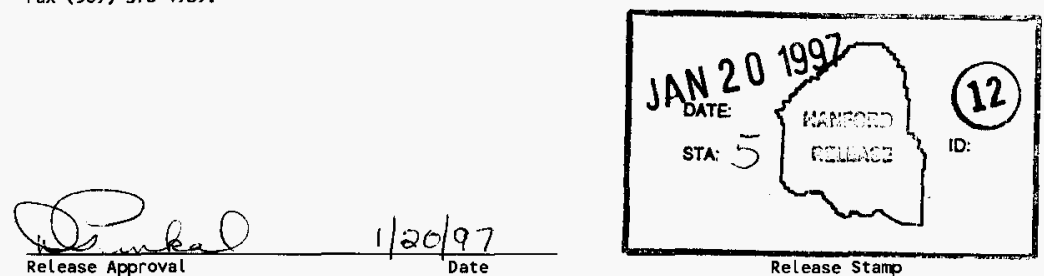

\section{Approved for Public Release}




$$
\text { HNF-SD-WM-RPT-287, Rev. } 0
$$

\subsection{DESCRIPTION OF EFFORT}

Approximately 2,000 drums of contact-handled (CH) transuranic (TRU) waste reside uncovered in trenches 1,20 , and 29 of burial ground $218-W-4 C$ in the 200 West Area. The U. S. Department of Energy intends, at some point, to transport all TRU waste to the Waste Isolation Pilot Plant near Carlsbad. New Mexico, for disposal. Therefore, wastes identified as TRU are expected to be retrieved from the burial grounds ${ }^{1}$.

There is concern that exposure to the elements may be significantly accelerating corrosion of the subject drums, complicating subsequent retrieval and handling. Relocation of the containers into covered storage would help to mitigate that concern.

In fiscal year 1996, an internal milestone was established to relocate approximately 250 of these containers into covered storage in the Central Waste Complex (CWC).

Through a review of available documentation and process knowledge, and selective screening. 260 candidate drums were identified for relocation. Based upon process knowledge, the drums were divided in five separate waste streams: 101A (4 drums). 102A (42 drums), 102B (27 drums), 103A (75 drums), and 103B (112 drums). A11 selected drums were in drum module 14 of trench 1 in burial ground $218-W-4 C$. The drums were located, inspected, and staged for transportation.

A representative number of drums (5\%) from each waste stream were transported to 224-T, the Transuranic Waste Storage and Assay Facility (TRUSAF). for non-destructive assay (NDA) and non-destructive examination (NDE). If no significant discrepancies were noted between the contents and the disposal records, the drums were considered to have passed verification. If $5 \%$ of a given waste stream passed verification, those drums were transported to the CWC and placed into storage, and the remainder of the drums in that waste stream were transported directly to the CWC from the burial grounds. If the initial 5\% failed verification, a second $5 \%$ was tested. If the second $5 \%$ failed, the entire waste stream was transported to 224-T for NDA/NDE.

Two waste streams, $101 \mathrm{~A}$ and $102 \mathrm{~B}$, passed verification on the first $5 \%$. One waste stream, 102A. failed verification of its first 5\%, but passed upon

1 Historical information regarding the placement of TRU wastes at Hanford is found in Contact-Handled Transuranic Waste Characterization Based on Existing Records. WHC-EP-0225. (WHC 1990). Regulatory issues are discussed in "Comprehensive Analysis of Regulatory Status and Alternate Contingencies for the Removal and Relocation of Transuranic Waste from Burial Ground 218-W4C. " a 1996 whitepaper produced by WHC Generator and Waste Acceptance Services. 
HNF-SD-WM-RPT-287, Rev. 0

successful verification of the second $5 \%$. The remaining two waste streams, $103 \mathrm{~A}$ and 103B. failed verification of both the fist and second $5 \%$ and required $100 \%$ verification.

Drums that did not pass verification could not be placed into storage unti 1 the issues were resolved. Some issues were resolved without opening the containers, by reclassifying the waste and overpacking if required. In some instances, however, visua 7 inspection of the drum contents was deemed appropriate. In such cases, the drum was staged in 224-T and then transported to T Plant for processing. There, following visual inspection of the contents, issues could be resolved by fixing the problem (e.g., removing prohibited articles), or by reclassification and/or overpacking.

Once verification issues were resolved, the container was staged, whether at 224-T or T Plant, and then transported to the CWC and placed into storage. The relocation effort is still in process.

A process flow chart from "Transuranic Waste Relocation Program Plan," WHC-SDWM-PAP-065, Rev. 0 (WHC 1996), is provided as Figure 1.

\subsection{SUMMARY OF CONTAINER SELECTION PROCESS}

Initially, the Solid Waste Information Tracking System (SWITS) database was used to screen for the most accessible drums. Approximately 500 containers were identified as either on or near the face of the drum modules. Then, the 500 drums were screened for:

- availability and completeness of process information, both documented and through staff interviews (to allow a determination regarding radiological and dangerous waste characteristics), and

- lot or batch size (using waste streams encompassing a large number of drums reduces the amount of process knowledge research needed and, in general, reduces the overa 11 number of drums requiring verification under the $5 \%$ standard).

Then. based upon a review of the obtained process knowledge, drums expected to contain mixed waste or prohibited articles were eliminated from consideration. Finally, drums containing $\geq 1 \mathrm{lg}$ fissile material were screened out, to comply with the T Plant waste acceptance criteria, facilitating its support in resolution of verification issues.

The screening process identified 260 candidate drums, a11 containing uncertified room wastes generated at the Plutonium Finishing Plant between October 1985 and June 1986. A11 260 drums were located in drum module 14, in trench 1 of burial ground 218-W-4C. Based upon similarities in process history. the 260 drums were divided into five waste streams, designated $101 \mathrm{~A}$ 
HNF-SD-WM-RPT-287, Rev. 0

(4 drums), 102A (42 drums), 102B (27 drums), 103A (75 drums), and 103B (112 drums).

\subsection{SUMMARY OF BURIAL GROUND ACTIVITY}

Drum retrieval operations, begun in mid August 1996, were conducted under work plan SW-WP-0120. Relocate Transuranic Waste from 218-W-4C Burial Ground.

Drum module 14 consisted of 55-gal1on drums placed in stacks 12 drums wide, 12 drums long, and up to 4 drums high, with plywood between each tier.

Initially, the work area and drum staging area were surveyed for radionuclides and toxic/hazardous substances per the Radiological Work Permit (RWP). Retrieval then consisted of the following steps, for each drum.

- locate the identified drum within the module and verify accuracy of SWITS location data

- $\quad$ survey accessible areas of drum for dose rate and smearable contamination

- determine if drum is safe to move by visually assessing drum integrity and verifying presence of vent clip

- move drum to inspection/staging area

- $\quad$ survey remaining areas of drum for dose rate and smearable contamination

- $\quad$ stage drum

- complete the visual inspection of drum

- complete Drum Inspection Form

- verify appropriate markings and labels are in place

The drum was then considered ready for transport.

Three drums were eliminated from consideration upon inspection. Each lacked a vent clip and was thus deemed unsafe for the purposes of this project; al 1 three were in waste stream 103B. All three remain in burial ground $218-\mathrm{W}-4 \mathrm{C}$. One was replaced in the drum module 14 of trench 1: the other two were relocated to trench 29 . Container relocation forms were prepared to document the relocation. 
HNF -SD-WM-RPT-287, Rev. 0

Retrieval necessitated moving a number of non-selected drums. These drums were staged, as needed, and placed back into the drum module. New locations were documented on container relocation forms.

The first $5 \%$ of each waste stream retrieved was transported to 224-T, the Transuranic Waste Storage and Assay Facility (TRUSAF), for assay and verification.

\subsection{SUMMARY OF ASSAY ACTIVITIES}

A significant portion of the TRU relocation containers were assayed at TRUSAF using the passive-active neutron assay machine. Of the 194 containers assayed. 31 exceeded the TRU $100 \mathrm{nCi} / \mathrm{g}$ limit and maintained their TRU designation. The remaining 163 containers (86\% of 194) were assayed at less than $100 \mathrm{nCi} / \mathrm{g}$ and therefore may be redesignated as low-level waste (L.LW). Successful verification results on these 163 containers would allow evaluation and eventual disposal of these containers in the burial grounds. Assay was conducted per SW-080-012, "Assay Transuranic Waste in 224-T."

Assay results are summarized in Table 1 . Container-specific assay results are provided in Attachment 1.

\subsection{SUMMARY OF VERIFICATION ACTIVITIES}

Successful verification of a representative sample of each waste stream is considered sufficient to allow placement of the remainder of that waste stream into storage. For wastes destined for the CWC, $5 \%$ is considered a representative sample, per the "Waste Analysis Plan for the Central Waste Complex" (WHC-SD-EN-WAP-003, Rev. 0) and the "Verification Program Marual for the Solid Waste Disposal Facilities" (WHC-SD-WM-WP-313. Rev. 0).

In addition, standard practice, implemented under the Performance Evaiuation System, allows that. should the first $5 \%$ fail verification, a second $5 \%$ can be tested. Successful verification of the second $5 \%$ would then allow the remainder of the waste stream to go directly to the CWC.

\subsection{RTR at TRUSAF}

192 drums were examined at $224-T$ using real-time radiography (RTR), an x-ray imaging and recording system that allows viewing of contents without opening the container. RTR was used to compare container contents with disposal records to look for discrepancies, to evaluate the integrity of internal secondary containment, and to screen for prohibited articles such as free liquids, caustics, lead, and pressurized containers. RTR was implemented under procedure SW-080-011. "Non-Destructive Examination of Solid Waste Drums at 224-T for Verification." 
HNF-SD-WM-RPT-287, Rev. 0

105 of the 192 containers passed verification without issue. The remaining 87 containers had verification issues of some sort. Verification results are summarized in Table 1. Verification issues are summarized in Table 2. Container-specific verification information is provided in Attachment 1.

\subsection{Issue Resolution at TRUSAF}

Verification issues that could be resolved without opening the containers were handled at 224-T. This type of resolution consisted of modifying the disposal record to reflect the "worst case" inventory, reclassifying the drum if required, and overpacking as needed. Issues with 47 of the 87 containers were resolved in this fashion at 224-T.

Nine of the drums were reclassified as caustic, typically due to the presence of batteries: one of these was also overpacked. 19 were reclassified as other mixed waste (OMW), usually due to the presence of lead: four of these were overpacked. 15 contained regulated non-hazardous wastes and were reclassified as non-mixed waste (NMW): eight of these were overpacked. Two had issues resolved by changing inventory records; no reclassification was necessary. Two had issues resolved simply by overpacking. One additional drum remains staged at 224-T, pending reclassification.

If it was necessary to open the container in order to resolve the verification issue, the drum was staged at 224-T and then transported to T Plant.

\subsection{Issue Resolution at T Plant}

To date, 35 have been transported to T Plant for resolution of verification issues. Four of the 35 have been processed, transported to the CWC and placed into storage. Ten have been processed, and wi 11 be transported when appropriate paperwork is completed. 21 drums are sti11 in process at T Plant. Five additional drums remain staged at 224- $T$, and may require processing at $T$ Plant.

T Plant opens and inspects the container contents, eliminating most uncertainty regarding inventory and secondary containment. If prohibited articles or regulated wastes are identified, they can either be removed, or the container can be reclassified. If secondary containment is an issue, the 7 iner can be properly closed, or the drum can be overpacked.

Documentation to date identified 13 containers that have passed verification at T Plant: 2 of these are still listed as in process. 7 await completion of paperwork. 4 are now in storage at the CWC.

15 containers are listed as having failed verification; 12 are still in process and 3 await completion of paperwork.

Verification results for seven containers at $T$ Plant are on hold.

January 20, 1997 


$$
\text { HNF-SD-WM-RPT-287, Rev. } 0
$$

\subsection{CONTAINER DISPOSITION}

Currently, 220 drums are in covered storage at the CWC, 31 drums remain at T Plant awaiting processing or completion of paperwork, and 6 drums remain staged at $224-\mathrm{T}$. 
HNF-SD-WM-RPT-287, Rev. 0

Table 1. Summary of Assay and Verification Results

\begin{tabular}{|c|c|c|c|c|c|c|}
\hline \multirow{2}{*}{$\begin{array}{l}\text { Waste } \\
\text { Stream }\end{array}$} & \multirow{2}{*}{$\begin{array}{l}\text { Verification } \\
\text { Drums }\end{array}$} & \multicolumn{2}{|c|}{ ASSAY } & \multicolumn{3}{|c|}{ VERIFICATION } \\
\hline & & $\mathrm{LLW}$ & TRU & Pass $^{\mathbf{A}}$ & Fail & Hold \\
\hline $101 \mathrm{~A}$ & 1 & 1 & 0 & 1 & 0 & 0 \\
\hline $102 A$ & 6 & 5 & 1 & 5 & 1 & 0 \\
\hline $102 B$ & 2 & $3^{B}$ & 0 & 2 & 0 & 0 \\
\hline $103 \mathrm{~A}$ & 75 & 62 & 13 & 67 & 3 & 5 \\
\hline $103 \mathrm{~B}$ & $109^{c}$ & 92 & 17 & 88 & 13 & 7 \\
\hline Total & 193 & 163 & 31 & 163 & 17 & 12 \\
\hline Percent & & $84 \%$ & $16 \%$ & $84 \%$ & $9 \%$ & $6 \%$ \\
\hline
\end{tabular}

A

60 of these drums had verification issues: 29 were redesignated mixed waste, 16 contained regulated wastes, 15 required overpacking.

B One non-verification drum was assayed

C No verification data available for PIN\# RHZ-103-A13927 
HNF-SD-WM-RPT-287, Rev. 0

\section{Table 2. Summary of Verification Issues}

\begin{tabular}{|c|c|c|}
\hline $\begin{array}{l}\text { Number } \\
\text { of } \\
\text { Drums }\end{array}$ & Percent ${ }^{A}$ & Issue \\
\hline 28 & $15 \%$ & contained liquids, either free or containerized \\
\hline 7 & $3 \%$ & $\begin{array}{l}\text { contained aerosol can or other pressurized } \\
\text { container }\end{array}$ \\
\hline 23 & $12 \%$ & contained lead (known or suspected) \\
\hline 12 & $6 \%$ & contained batteries (known or suspected) \\
\hline $29^{8}$ & $15 \%$ & $\begin{array}{l}\text { designated mixed waste ( } 13 \text { lead. } 9 \text { battery. } 3 \\
\text { light bulb) }\end{array}$ \\
\hline 6 & $3 \%$ & contained mask filters or HEPA filters \\
\hline 16 & $8 \%$ & $\begin{array}{l}\text { required overpacking due to presence of free } \\
\text { liquids or due to improper secondary containment } \\
\text { (no "horse tail" on the plastic lirier) }\end{array}$ \\
\hline 16 & $8 \%$ & $\begin{array}{l}\text { contained regulated waste; requires chemical } \\
\text { analysis prior to disposal }\end{array}$ \\
\hline
\end{tabular}

A Percentage of the 192 drums that went through the verification process. Note that several drums fall into more than one category.

B To date, 39 drums, $20 \%$ of the 192 verified drums, have been identified as mixed waste. Only 29 have actually been reclassified as mixed waste. Of the remaining ten drums, two had the dangerous waste removed at T-Plant, six remain in process at T-Plant, and two remain staged at 224-T. 
HNF-SD-WM-RPT-287, Rev. 0

Figure 1. Relocation Process Flowchart

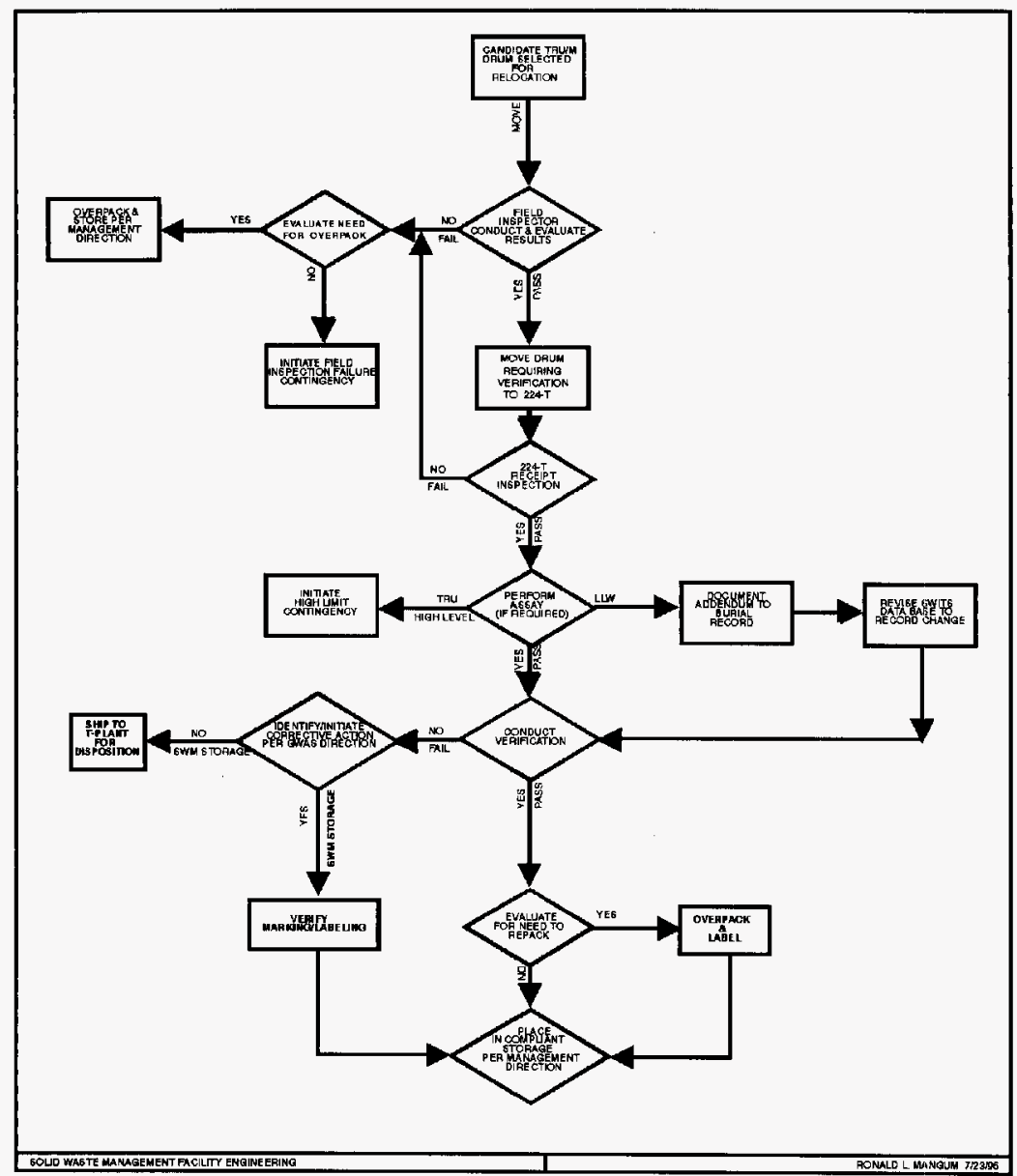

January 20, 1997 
Container-Specific Summary

\begin{tabular}{|c|c|c|c|c|}
\hline PIN\# & STREAM & ASSAY ISSUE & STATUS CONHENTS & LOCRTTON \\
\hline RHZ-101-A13637 & $101 \mathrm{~A}$ & & & CWC \\
\hline RHZ-101-A13707 & $101 \mathrm{~A}$ & & & CWC \\
\hline RHZ-101-A13708 & $101 \mathrm{~A}$ & & & CWNC \\
\hline RHZ-101-A14105 & $101 \mathrm{~A}$ & UW & Pass & CMV \\
\hline RHZ-102-A13626 & $102 A$ & & & Cre \\
\hline RHZ-102-A13672 & $102 \mathrm{~A}$ & & & CWC \\
\hline RHZ-102-A13673 & $102 \mathrm{~A}$ & & & CWC \\
\hline RHZ-102-A13687 & 102A & & & CWC \\
\hline RHZ-102-A13688 & $102 \mathrm{~A}$ & & & ENS \\
\hline RHZ-102-A13689 & $102 A$ & & & CNKC \\
\hline RHZ-102-A13690 & $102 \mathrm{~A}$ & & & Cunc \\
\hline$R H Z-102-A 13691$ & $102 \mathrm{~A}$ & & & CMC \\
\hline RHZ-102-A13692 & $102 A$ & & & che \\
\hline RHZ-102-A13763 & 102A & & & CWC \\
\hline RHZ-102-A13766 & 1024 & & & CWC \\
\hline RHZ-102-A13768 & $102 \mathrm{~A}$ & & & CWV \\
\hline RHZ-102-A13921 & $102 \mathrm{~A}$ & & & CWVC \\
\hline RHZ-102-A13962. & $102 \mathrm{~A}$ & & & Cur \\
\hline $\mathrm{RHZ}-102-\mathrm{A} 13966$ & $102 \mathrm{~A}$ & & & CNic \\
\hline RHZ-102-A14070 & $102 \mathrm{~A}$ & & & CMC \\
\hline RHZ-102-A14112 & $102 \mathrm{~A}$ & & & CWNC \\
\hline RHZ-102-A14263 & $102 \mathrm{~A}$ & & & CVMC \\
\hline RHZ-102-A14264 & $102 \mathrm{~A}$ & & & CNC \\
\hline RHZ-102-A14461 & $102 \mathrm{~A}$ & & & GMV \\
\hline RHZ-102-A.14788 & $102 \mathrm{~A}$ & & & CWN \\
\hline RHZ-102-A14789 & $102 \mathrm{~A}$ & LLW & Pass & CMC \\
\hline RHZ-102-A14790 & $102 \mathrm{~A}$ & & & CWM \\
\hline RHZ-102-A14791 & $102 \mathrm{~A}$ & LW Excessive Free liquid & TO PROGESS & T-Plant \\
\hline $\mathrm{RHZ}-102-A 14792$ & $102 \mathrm{~A}$ & LLW & Pass & CWKS \\
\hline
\end{tabular}

OMW Other Mised Viaste

NMW Non-Mixed Waste

op Overpacked 
Attachment 1

\begin{tabular}{|c|c|c|c|c|}
\hline PIN\# & STREAM & ASSAY ISSUE & STATUS CONMENTS & LOCATION \\
\hline RHZ-102-A14793 & $102 A$ & & & CWK \\
\hline RHZ-102-A14794 & $102 \mathrm{~A}$ & LLW & Pass & $\mathrm{CWC}$ \\
\hline RHZ-102-A14838 & $102 \mathrm{~A}$ & LLW & Pass & CWNE \\
\hline RHZ-102-A14839 & $102 \mathrm{~A}$ & TRU & Pass & CWK \\
\hline RHZ-105-A13575 & $102 \mathrm{~A}$ & & & CINC \\
\hline $\mathrm{RHZ}-108-\mathrm{A} 13648$ & $102 \mathrm{~A}$ & & & CWM \\
\hline RHZ-108-A13649 & $102 \mathrm{~A}$ & & & CWVC \\
\hline RHZ=108-A13669 & $102 \mathrm{~A}$ & & & CWN \\
\hline RHZ-108-A13670 & $102 \mathrm{~A}$ & & & CWN \\
\hline RHZ-108-A13671 & 102A & & & CWC \\
\hline RHZ-108-A13674 & $102 \mathrm{~A}$ & & & CWC \\
\hline RHZ-108-A13675 & $102 \mathrm{~A}$ & & & CWC \\
\hline RHZ-109-A13680 & $102 A$ & & & CWC \\
\hline RHZ-109-A13681 & $102 \mathrm{~A}$ & & & CWR \\
\hline RHZ-109-A13682 & $102 \mathrm{~A}$ & & & CWC \\
\hline RHZ-109-A13683 & $102 A$ & & & CWR \\
\hline RHZ-109-A13684 & $102 A$ & & & CYK \\
\hline RHZ-102-A13847 & $102 B$ & & & CIMC \\
\hline $\mathrm{RHZ}-102-\mathrm{A} 13850$ & $102 B$ & & & CNK \\
\hline RHZ-102-A13913 & $102 B$ & & & CWC \\
\hline RHZ-102-A13914 & $102 \mathrm{~B}$ & & & CWve \\
\hline RHZ-102-A13922 & $102 B$ & & & CNAC \\
\hline RHZ-102-A13961 & 1028 & & & CMC \\
\hline RHZ-102-A13963 & $102 B$ & & & CWV \\
\hline RHZ-102-A13964 & 1028 & & & CWC \\
\hline RHZ-102-A13965 & $102 \mathrm{~B}$ & & & CWE \\
\hline RHZ-102-A13967 & $102 B$ & & & $\mathrm{CWN}$ \\
\hline RHZ-102-A13971 & 1028 & & & CWN \\
\hline $\mathrm{RHZ}-102-\mathrm{A} 13972$ & $102 B$ & & & CWV \\
\hline
\end{tabular}

OMW Other Mixed Waste

NMW Non-Mixed Waste

OP Overpacked 
Attachment 1

Container-Specific Summary

\begin{tabular}{|c|c|c|c|c|}
\hline PIN \# & STREAM & ASSAY ISSUE & STATUS COMMENTS & LOCATION \\
\hline RHZ-102-A13973 & $102 \mathrm{~B}$ & & & Che \\
\hline RHZ-102-A14021 & $102 \mathrm{~B}$ & & & CMC \\
\hline RHZ-102-A14040 & $102 \mathrm{~B}$ & & & CWE \\
\hline RHZ-102-A14042 & $102 \mathrm{~B}$ & & & CWM \\
\hline RHZ-102-A14046 & $102 \mathrm{~B}$ & LLM & & CWK \\
\hline RHZ-102-A14064 & 1028 & & & CWV \\
\hline RHZ-102-A14067 & 1028 & & & CWV \\
\hline $\mathrm{RHZ}-102-A 14078$ & $102 B$ & LLW n/a & Pass & Che \\
\hline $\mathrm{RHZ}-102-A 14079$ & 1028 & & & CWC \\
\hline RHZ-102-A14106 & $102 B$ & & & CWC \\
\hline RHZ-102-A14107 & $102 B$ & & & CHAC \\
\hline $\mathrm{RHZ}-102-A 14108$ & $102 \mathrm{~B}$ & & & CME \\
\hline Fi1Z-102-A14109 & $102 B$ & LLV n/a & Pass & CWK \\
\hline RHZ-102-A14111 & $102 \mathrm{~B}$ & & & OVIC \\
\hline $\mathrm{RHZ}-102-\mathrm{A} 14113$ & 1028 & & & CWP \\
\hline RHZ-103-A13584 & $103 A$ & LWW & Pass & CWC \\
\hline$R H \overline{Z-103-A 13586}$ & $10 \overline{3 A}$ & LLW Containerized Liquid & TO PROCESS & T-P tant \\
\hline RHZ-103-A13793 & $103 \mathrm{~A}$ & TRU Inventory. Check on MSA/Combo Filters & NAYU-OVERPACK & CWC \\
\hline RHZ-103-A13795 & $103 A$ & LLV & Pass & CWV \\
\hline RHZ-103-A13B19 & $103 \mathrm{~A}$ & LLW & Pass & CWV \\
\hline RHZ-103-A13820 & $103 A$ & LLW & Pass & CWK \\
\hline RHZ-103-A13821 & $103 \mathrm{~A}$ & LLW & Pass & CWN \\
\hline RHZ-103-A13822 & $103 \mathrm{~A}$ & LLW & Pass & Cnk \\
\hline RHZ-103-A13823 & $103 \mathrm{~A}$ & LLW & Pass & CWC \\
\hline RHZ-103-A 13825 & $103 \mathrm{~A}$ & LLW & Pass & CWC \\
\hline$R H Z-103-A 13826$ & $103 \mathrm{~A}$ & LLW & Pass & CWE \\
\hline RHZ-103-A13853 & $103 \mathrm{~A}$ & LLW & Pass & CWC \\
\hline RHZ-103-A13855 & $103 \mathrm{~A}$ & LLW & Pass & CNS \\
\hline $\mathrm{RHZ}-103-\mathrm{A13898}$ & $103 \mathrm{~A}$ & TRU & Pass & EWV \\
\hline
\end{tabular}

OMIW Other Mixed Waste

NMW Non-Mixed Wraste

OP Overpacke: 
Attachment 1

Container-Specific Summary

\begin{tabular}{|c|c|c|c|c|c|c|}
\hline PIN\# & STREAM & ASSAY & TSSUE & STATUS & COMMENTS & LOCATION \\
\hline RHZ-103-A13899 & $103 \mathrm{~A}$ & LLW & $>1 \%$ free liquid & Pass & NMW-OVERPACK & CWC \\
\hline RHZ-103-A13904 & $103 \mathrm{~A}$ & LLW & Lead article suspect shavings & Pass & OMW & CWC \\
\hline RHZ-103-A13917 & $103 \mathrm{~A}$ & LLW & & Pass & & CWC \\
\hline RHZ-103-A13919 & $103 \mathrm{~A}$ & $\underline{L W}$ & Free liquid and suspect lead & Fail* & TO PROCESS & T-Plant \\
\hline RHIZ-103-A14233 & $103 \mathrm{~A}$ & LWW & & Pass & & che \\
\hline RHZ-103-A14276 & $103 \mathrm{~A}$ & LLW & & Pass & & CWC \\
\hline RHZ-103-A14277 & $103 A$ & LLW & containerized liquids & Hold & TO PROCESS & T-Plant \\
\hline $\mathrm{RHZ}-103-\mathrm{A} 14280$ & $103 A$ & TRU & & Pass & & CMNC \\
\hline RHZ-103-A14281 & $103 A$ & LLW & & Pass & & CMC \\
\hline RHZ-103-A14288 & $\overline{103 A}$ & LLW & Inventory Changes & Pass & NMW & SWC. \\
\hline RHZ-103-A14289 & $103 \mathrm{~A}$ & LIW & GM or PAM w/balteries Incorrect Inventory & Pass & CAUSTIC & CWC \\
\hline RHZ-103-A14292 & 103A & TRU & Suspect Hy. Ram Oil & Fail & TOPROCESS & T-Plant \\
\hline RHZ-103-A14293 & $103 \mathrm{~A}$ & LLW & Suspect Lead Shavings & Pass & ONW & CMC \\
\hline $\mathrm{RHZ}-103-\mathrm{A} 14437$ & $103 \mathrm{~A}$ & LLW & & Pass & & CWC \\
\hline $\mathrm{RHZ}-103-\mathrm{A} 14438$ & $103 \mathrm{~A}$ & LWW & & Pass & & CWC \\
\hline RHZ-103-A14439 & $103 A$ & LLW & Containerized Liquid & Pass & TOPROCESS & T-Plant \\
\hline RHZ-103-A14450 & $103 A$ & LLW & Aerosol Can & Hold & TO PROCESS & T-Plant \\
\hline RHZ-103-A14451 & $103 A$ & IIW & Light Buths & Pass & OMW & CWC \\
\hline RHZ-103-A14452 & $103 \mathrm{~A}$ & LLW & Containerized liquid, suspect $\mathrm{Pb}$ Brick/Spill Pallet & Hold & TOPROCESS & T-Plant \\
\hline RHZ-103-A14453 & $103 \mathrm{~A}$ & TRU & Suspect Pb Brick & Pass & ONW-OVERPACK & CWC \\
\hline RHZ-103-A14454 & $103 \mathrm{~A}$ & LLW & & Pass & & CWK \\
\hline RHZ-103-A14801 & $103 A$ & LLW & & Pass & & CWC \\
\hline RHZ-103-A14802 & $103 \mathrm{~A}$ & TRU & & Pass & & CWC \\
\hline RHZ-103-A14803 & $103 \mathrm{~A}$ & LLW & & Pass & & CWK \\
\hline RHZ-103-A14804 & $103 \mathrm{~A}$ & LLW & & Pass & & CWK \\
\hline RHZ-103-A14805 & $103 \mathrm{~A}$ & TRU & Misc "Colorimetric" tubes & Hold & TO PROCESS & T-Plant \\
\hline RHZ-103-A14806 & $103 \mathrm{~A}$ & TRU & & Pass & & CNC \\
\hline $\mathrm{RHZ}-103-\mathrm{A14807}$ & $103 \mathrm{~A}$ & TRU & & Pass & & CWNC \\
\hline $\mathrm{RHZ}-103-A 14808$ & $103 \mathrm{~A}$ & TRU & Battery, circuit board & Pass & CAUSTIC-OP & CWVC \\
\hline
\end{tabular}

OMW Other Mxed Waste

NMW Non-Mixed Waste

OP Overpacked 
Attachment 1

HNF-SD-WM-RPT-287, Rev, 0

Container-Specific Summary

\begin{tabular}{|c|c|c|c|c|c|}
\hline PIN \# & STREAM & ASSAY ISSUE & STATUS & COMMENTS & LOCATION \\
\hline RHZ-103-A14809 & $103 \mathrm{~A}$ & LLW & Pass & & CMC \\
\hline RHZ-103-A $\$ 4810$ & $103 A$ & LLW & Pass & & Garc \\
\hline RHZ-103-A14822 & $103 A$ & LLW Lead/Removed & Pass & & CMC \\
\hline $\mathrm{RHZ}-103-\mathrm{A14823}$ & $103 \mathrm{~A}$ & TRU 2 "d" batteries 1 gv partial bttle wht out/ & Pass & CAISTIC & CWC \\
\hline RHZ-103-A14824 & $103 \mathrm{~A}$ & LLW & Pass & & $\mathrm{CVIC}$ \\
\hline RHZ-103-A14825 & $103 A$ & LLW & Pass & & CWVC \\
\hline RHZ-103-A14830 & $103 A$ & LLW & Pass & & CWKC \\
\hline RHZ-103-A14831 & $103 \bar{A}$ & LLW Light Bulbs/No ong. papers? & Hold & TO PROCESS & T-Plant \\
\hline RHZ-103-A14832 & $103 \mathrm{~A}$ & LLW & Pass & & CMc \\
\hline RHZ-103-A14833 & $103 \mathrm{~A}$ & LLW & Pass & & Give \\
\hline RHZ-103-A14834 & $103 \mathrm{~A}$ & LLW & Pass & & CNC \\
\hline RHZ-103-A14845 & $103 \mathrm{~A}$ & Compare to A13812 Fluorescent Starters & Pass & NMWUOVERPACK & CWN \\
\hline$R H Z 2-103-A 14040$ & $103 \dot{A}$ & TRU & Pass & & CWC \\
\hline RHZ-103-A14847 & $103 \mathrm{~A}$ & $>1 \%$ Free Liquid & Pass & PAPERWORK & T-PIani \\
\hline RHZ-103-A14B48 & $103 \mathrm{~A}$ & Mercuny Clean-up & Pass & PAPERWWORK & T-Fiant \\
\hline RHZ-103-A14849 & $103 \mathrm{~A}$ & LLW & Pass & & CWV \\
\hline RHZ-103-A14850 & $103 \mathrm{~A}$ & LLW & Pass & & GWC \\
\hline RHZ-103-A14851 & $103 \mathrm{~A}$ & Inventory Changes & Pass & & onc \\
\hline RHZ-103-A14852 & $103 \mathrm{~A}$ & Inventory changes required & Pass & NMWOOVERPACK & CMC \\
\hline$R H Z-103-A 14855$ & $103 \mathrm{~A}$ & LLW & Pass & & CWC \\
\hline RHZ-103-A14856 & $103 \mathrm{~A}$ & Light bulbiremoved & Pass & PAPERWORK & T-Plant \\
\hline RHZ-103-A14858 & $103 \mathrm{~A}$ & LLW & Pass & & CWK \\
\hline $\mathrm{RHZ}-103-\mathrm{A} 14863$ & $103 A$ & LLW & Pass & & clve \\
\hline $\mathrm{RHZ}-103-\mathrm{A} 14864$ & $103 \mathrm{~A}$ & LLW & Pass & & ChK \\
\hline RHZ-103-A14865 & $103 \mathrm{~A}$ & LLW & Pass & & chre \\
\hline RHZ-103-A14866 & $103 A$ & Containerized liquids & Fail & TOPROCESS & T-Plant \\
\hline RHZ-103-A14891 & $103 A$ & LIW & Pass & & cive \\
\hline RHZ-103-A14892 & $103 \mathrm{~A}$ & LLW & Pass & & CWC \\
\hline RHZ-103-A14893 & $103 \mathrm{~A}$ & TRU & Pass & & CWC \\
\hline
\end{tabular}

OMW Other Mixed Maste

NHW Non-Mixed Waste

OP Overpacked 


\begin{tabular}{|c|c|c|c|c|c|c|}
\hline PlN \# & STREAM & ASSAY & ISSUE & STATUS & COMMENTS & LQCATION \\
\hline RHZ-103-A14894 & $103 A$ & LLW & & Pass & & CWM \\
\hline RHZ-103-A14895 & $103 \mathrm{~A}$ & LIW & Aerosol Can (Pressurized) ?? & Pass & PAPERWORK & T-Pant \\
\hline RHZ-103-A14896 & $103 \mathrm{~A}$ & LEW & & Pass & & CHC \\
\hline RHZ-103-A13587 & $103 \mathrm{~B}$ & TRU & Battery & Pass & CAUSTIC & CAM \\
\hline $\mathrm{RHZ}-103-A 13588$ & 1038 & LLW & & Pass & & CHC \\
\hline RHZ-103-A13589 & $103 B$ & LLW & Containerized Liquid & Hold & TOPROCESS & T-Plant \\
\hline $\mathrm{RHZ}-103-\mathrm{A} 13595$ & 1038 & LLW & & Pass & & CWC \\
\hline RHZ-103-A13597 & $103 B$ & LLW & light bulb & Pass & ONN & CWV \\
\hline RHZ-103-A13598 & $103 B$ & LLW & 2 possible lead sheet pieces & Pass & OMW & CWK \\
\hline RHZ-103-A13643 & $103 B$ & TRU & Containerized Liquid & Fail & TO PROCESS & T-Plant \\
\hline RHZ-103-A13644 & $103 B$ & TRU & Suspect Lead & Pass & OMHA-OMERPACK & CHC \\
\hline RHZ-103-A13645 & $103 B$ & LLW & Liquid Type & Pass & OVERPACK & CWV \\
\hline RHZZ-103-A13646 & 1038 & Lin & Containerized Liquid & Fail & TO PROCESS & T-Plant \\
\hline RHZ-103-A13647 & $103 \mathrm{~B}$ & LLW & & Pass & & CWV \\
\hline RHZ-103-A13677 & 1038 & LLY & $>1 \%$ Frea Liquid & Pass & PAPERMORK & T-Plant \\
\hline RHZ-103-A13678 & 1038 & TRU & & Pass & & CWNC \\
\hline RHZ-103-A13679 & $103 \mathrm{~B}$ & LLW & Lead Containing article (light bulb) & Pass & ONIN & CWC \\
\hline RHZ-103-A13685 & $103 B$ & LLW & & Pass & & CHac \\
\hline RHZ-103-A13686 & $103 \mathrm{~B}$ & LLW & Suspect Lead Solder & Pass & OMN & CHAC \\
\hline $\mathrm{RHZ}-103-\mathrm{A} 13697$ & $103 B$ & LLW & & Pass & & CWK \\
\hline RHZ-103-A13698 & $103 B$ & TRU & Lead shielding & Pass & OMN-OVERPACK & CWC \\
\hline RHZ-103-A13699 & $103 B$ & LLW & Lead strongly suspected & Pass & OMM-OVERPACK & CWC \\
\hline RHZ-103-A13700 & $103 B$ & LLW & & Pass & & CWN \\
\hline RHZ-103-A13701 & 1038 & LLW & & Pass & & CWC \\
\hline $\mathrm{RHZ}-103-\mathrm{A} 13702$ & $103 B$ & TRU & & Pass & & $\mathrm{CWC}$ \\
\hline $\mathrm{RHZ}-103-\mathrm{A} 13703$ & $103 B$ & TRU & & Pass & & $\mathrm{CWC}$ \\
\hline RHZ-103-A13704 & $103 B$ & LLW & & Pass & & CWC \\
\hline RHZ-103-A13706 & 1038 & LLW & & Pass & & CWV \\
\hline RHZ-103-A13709 & $103 B$ & & NO VENT CLIP & & & LLBG \\
\hline
\end{tabular}

OMW Other Mixeo Waste

NMW Non-Mixed Waste

OP Overpacked 
Container-Specific Summary

\begin{tabular}{|c|c|c|c|c|c|}
\hline PIN \# & STREAM & ASSAY ISSUE & STATUS & COMMENTS & LOCATION \\
\hline RHZ-103-A13710 & 1038 & LLW & Pass & & CWD \\
\hline RHZ-103-A13712 & $103 B$ & LLW & Pass & & CWRC \\
\hline RHZ-103-A13713 & $103 \mathrm{~B}$ & LEWW & Pass & & CNMC \\
\hline RHZ-103-A13714 & 1038 & LEWV & Pass & & CWM \\
\hline RHZ-103-A13715 & $103 B$ & NO VENT CLIP & & & LLBG \\
\hline RHZ-103-A13716 & $103 B$ & Battery & Pass & CAUSTIC & CMC \\
\hline $\mathrm{RHZ}-103-\mathrm{A} 13717$ & $103 B$ & Inventory & Pass & NHWLOVERPACK & CWC \\
\hline RHZ-103-A13718 & $103 \mathrm{~B}$ & Aerosol, Battery, lead, containerized liquid & Fail* & TO PROCESS & T-Plant \\
\hline RHZ-103-Ā13719 & $103 B$ & (2) & Pass & & CWK \\
\hline RHZ-103-A13720 & $103 B$ & LLW & Pass & & CWC \\
\hline RHZ-103-A13721 & $103 B$ & LLW & Pass & & CWM \\
\hline RHZ-103-A13725 & 1038 & Batteries & Pass & CAUSTIC & Chr \\
\hline RHZ-103-A13726 & 1030 & inventory Fixes & Pass & NMW & CWC \\
\hline RHZ-103-A13727 & $103 B$ & 6"D" Batteries & Pass & CAUSTIC & CrrC \\
\hline $\mathrm{RHZ}-103-\mathrm{A} 13728$ & $103 B$ & Inventory Changes & Pass & & CNV \\
\hline RHZ-103-A13775 & $103 B$ & 9volt Battery & Pass & CAUSTIC & EVHe \\
\hline RHZ-103-A13777 & $103 B$ & Hepa Filker status & Hold" & & $224 \mathrm{~T}$ \\
\hline RHZ-103-A13778 & $103 B$ & TRU & Pass & & cutc \\
\hline RHZ-103-A13779 & $103 \bar{B}$ & Combo. Fitters (3) & Pass & NMWN & CWC \\
\hline RHZ-103-A13781 & $103 B$ & Containerized Liquids, poss lead, aerosol can & Fail & TOPROCESS & T-Plant \\
\hline RHZ-103-A13783 & $103 B$ & Lead containing articles (fuses) & Pass & ONW & CWE \\
\hline RHZ-103-A13784 & $103 B$ & LLVW & Pass & & CWC \\
\hline RHZ-103-A13785 & $103 \mathrm{~B}$ & Free Liquids & Pass & NMW-OVERPACK & CWC \\
\hline $\mathrm{RHZ}-103-\mathrm{A} 13786$ & $103 \bar{B}$ & LLW & Pass & & CWV \\
\hline RHZ-103 A13791 & $103 \mathrm{~B}$ & $>1 \%$ Free Liquid & Fail* $^{*}$ & OVERPACK & $\mathrm{CMC}$ \\
\hline $\mathrm{RHZ}-103-\mathrm{A} 13792$ & $103 \mathrm{~B}$ & LLW & Pass & & CMC \\
\hline RHZ-103-A13801 & 1038 & LLW & Pass & & $\mathrm{CWC}$ \\
\hline RHZ-103-A13802 & $103 \mathrm{~B}$ & Inventory Fixes & Pass & NMWN & CVre \\
\hline$R H Z-103-A 13803$ & $103 B$ & Containerized Liquid/Possible Battery & Fail & TO PROCESS & T-Plant \\
\hline
\end{tabular}

OWW Other Mixed Waste

NMW Non-Mxed Waste

OP Overpacked 
Attachment 1

\begin{tabular}{|c|c|c|c|c|c|c|}
\hline PIN \# & STREAM & ASSAY & ISSUE & STATUS & COMMENTS & LOCATION \\
\hline RHZ-103-A13804 & 1038 & LLW & Inventory Fixes & Pass & NMW & CWK \\
\hline RHZ-103-A13805 & $103 \theta$ & LLW & Possible Lead & Hold & & $224 \mathrm{~T}$ \\
\hline RHZ-103-A13806 & 1038 & LLW & Containerized Liquids & Fail & TOPROCESS & T-Plant \\
\hline RHZ-103-A13807 & $103 \mathrm{~B}$ & LLW & Containerized Liquid & Fail & TOPROCESS & T-Plant \\
\hline RHZ-103-A13808 & 1038 & LLW & & Pass & & CWNC \\
\hline RHZ-103-A13808 & 1038 & LLW & Inventory changes & Pass & NMW & CWNC \\
\hline $\mathrm{RHZ}-103-\mathrm{A} 13812$ & $103 B$ & LLW & Light bulb, battery, wristwatch, syringe wiliquid & Fail ${ }^{*}$ & & $224 \mathrm{~T}$ \\
\hline RHZZ-103-A13814 & $103 B$ & LLW & & Fail & TO PROCESS & T-Plant \\
\hline $\mathrm{RHZ}-103-\mathrm{A13815}$ & $103 \mathrm{~B}$ & LLW & & Pass & & CWC \\
\hline RHZ-103-A13816 & $103 \mathrm{~B}$ & LLW & Scott Brand Mask Fitter & Pass & NMW & CWC \\
\hline RHZ-103-A13B17 & $103 \mathrm{~B}$ & LLW & Cincut Brd (containing lead) & Pass & OMW & CWC \\
\hline RHZ-103-A13B18 & 1038 & LLW & & Pass & & CWNC \\
\hline $\mathrm{RHZ}-103-\mathrm{A} 13824$ & 1038 & LLW & & Pass & & CUNC \\
\hline $\mathrm{RHZ}-103-\mathrm{A} 13827$ & 1038 & LLW & & Pass & & CYNC \\
\hline RHZ-103-A13828 & 103B & LLW & Lead Disc (approx. 6") & Pass & OMN & CWW \\
\hline RHZ-103-A13830 & 103E & LLW & & Pass & & CWN \\
\hline RHZ-103-A13831 & 103B & LLW & & Pass & & CWE \\
\hline RHYZ-103-A13834 & $103 \mathrm{~B}$ & LLW & combination mask filters & Hold* & & $224 T$ \\
\hline RHZ-103-A13835 & 103B & LLW & Aerosol can (approx. 3 to 4 oz.) & Fail & PAPERWORK & T-Plant \\
\hline RHZ-103-A13836 & $103 \mathrm{~B}$ & & NO VENT CLIP & & & LLEG \\
\hline $\mathrm{RHZ}-103-\mathrm{A} 13837$ & $103 \mathrm{~B}$ & LLW & possible pressurized container & Faj** & PAPERYIORK & T-Plant \\
\hline RHZ-103-A13838 & $103 \mathrm{~B}$ & LLW & Light bulb & Pass & ONW & GNC \\
\hline RHZ-103-A13859 & 1038 & LLW & & Pass & & CWC \\
\hline RHZ-103-A13860 & 1038 & LLW & NOTE: Metal $50 \%$ & Pass & & CWV \\
\hline RHZ-103-A13861 & 1038 & LLW & & Pass & & CMV \\
\hline RHZ-103-A13862 & $103 \mathrm{~B}$ & LLW & Approx. 2x5" lead & Pass & OMW & CMN \\
\hline RHZ-103-A13923 & $103 \mathrm{~B}$ & TRU & & Pass & & CWNC \\
\hline RHZ-103-A13926 & $103 \mathrm{~B}$ & LLW & & Pass & & CMC \\
\hline $\mathrm{RHZ}-103-\mathrm{A} 13927$ & $103 \mathrm{~B}$ & LLW & & & & CWNC \\
\hline
\end{tabular}

OMW Other Mixed Waste

NMW Non-Mixed Waste

OP Overpacked 
Container-Specific Summany

\begin{tabular}{|c|c|c|c|c|c|c|}
\hline PIN & STREAM & ASSAY & ISSUE & STATUS & COMMENTS & LOCATION \\
\hline $\mathrm{RHZ}-103-\mathrm{A} 13929$ & 1038 & LLW & Aerosol can (no puncture/press??) & Fail & PAPERWORK & T-Plant \\
\hline RHZ-103-A13930 & $103 \mathrm{~B}$ & LLW & & Pass & - & CWC \\
\hline RHZ-103-A13940 & $103 B$ & LLW & & Pass & & CWV \\
\hline RHZ-103-A13941 & 1038 & TRU & Possible Battery & Pass & CAUSTIC & CHK \\
\hline RHZ-103-A13946 & $103 B$ & LLW & & Pass & & CWM \\
\hline RHZ-103-A13949 & $103 B$ & LLW & $>1 \%$ free liquid & Pass & NAW-OVERPACK & GWC \\
\hline RHZ-103-A13951 & $103 \mathrm{~B}$ & LLW & Containerized Liquids & Pass & PAPERWORK & T-Plant \\
\hline RHZ-103-A13952 & $103 B$ & LLW & Free liquid $>1 \%$ (approx. $11 / 2$ gallon) & Pass & NWWOVERPACK & CWC \\
\hline RHZ-103-A13954 & $103 B$ & TRU & & Pass & & CWC \\
\hline RHZ-103-A13958 & $103 \mathrm{~B}$ & LLW & OlL? & Hold" & & $224 T$ \\
\hline RHZ-103-A14016 & $103 \mathrm{~B}$ & LLW & $>1 \%$ Free Liquids & Pass & NMW-OYERPACK & CHC \\
\hline RHZ-103-A14018 & $103 \mathrm{~B}$ & LLW & Suspect Lead Shavings & Pass & ONH & CMK \\
\hline RHZ-103-A14237 & 1038 & LLW & & Pass & & CVMC \\
\hline $\mathrm{RHZ}-103-\mathrm{A} 14238$ & $103 B$ & TRU & Containerized liquid & Hold & TOPROCESS & T-Plant \\
\hline RHZ-103-A14239 & $10 \overline{B B}$ & LLW & Lead Bricks & Pass & OAHA & CWC \\
\hline RHZ-103-A14240 & $103 \mathrm{~B}$ & LLW & Suspect Lead Flakes/Chips & Pass & OMVN & CWC \\
\hline RHZ-103-A14241 & $103 \mathrm{~B}$ & LLW & & Pass & & CWC \\
\hline RHZ-103-A14242 & $103 B$ & LLW & & Pass & & CWE \\
\hline RHZ-103-A1427B & $103 B$ & LLW & & Pass & & CWC \\
\hline RHZ-103-A14279 & $103 \mathrm{~B}$ & TRU & & Pass & & CWE \\
\hline RHZ-103-A14287 & 1038 & LLW & Suspect lead & Pass & OMW & CWYC \\
\hline RHZ-103-A14290 & $103 \mathrm{~B}$ & LLW & Hepa Filter & Hold" & & $224 \mathrm{~T}$ \\
\hline RHZ-103-A14441 & $103 B$ & LLW & & Pass & & Sve \\
\hline RHZ-103-A 14442 & 103B & LLW & & Pass & & CWNC \\
\hline $\mathrm{RHZ}-103-\mathrm{A} 14826$ & 103B & LLW & & Pass & & CWC \\
\hline RHZ-103-A14827 & $103 B$ & LLW & & Pass & & CWC \\
\hline RHZ-103-A14828 & 103B & LLW & & Pass & & CWC \\
\hline RHZ-103-A14829 & $103 \bar{B}$ & LLW & Containerized liquid/Removed & Pass & PAPERWORK & T-Plant \\
\hline
\end{tabular}

OMW Other Mixed Waste

NNW Non-Mixed Waste

aP Overpacked 